\section{Etiquetado de alimentos en Ecuador}

Hemos leído la reciente publicación de Díaz y colaboradores "Etiquetado de alimentos en Ecuador: implementación, resultados y acciones pendientes" publicado en Rev Panam Salud Publica. 2017;41:e54 (1). El reporte "analiza el proceso de implementación del etiquetado de alimentos procesados, los resultados alcanzados hasta la fecha y propone medidas complementarias ..."(1).

Los datos que se reportan tienen limitaciones que no se indican en el manuscrito. Por ejemplo, los datos utilizados en la publicación son datos secundarios obtenidos de entrevistas y de estudios de mercado (en la sección de Materiales y Métodos no se indican detalles de instrumentos utilizados ni de su validez). Además, los datos reportados, en su mayoría cualitativos, no profundizan en aspectos fundamentales que indiquen el valor real del etiquetado nutricional tipo semáforo (ES) en la compra de los alimentos; se indica que "El semáforo es ampliamente reconocido y comprendido por parte de los consumidores", sin embargo, no se analiza en profundidad el nivel de entendimiento que los individuos tienen al utilizar el ES y cómo esto afecta en la elección de alimentos saludables (¿qué significan los colores desde el punto de vista nutricional?). Si los autores consideraran al ES como un instrumento ideal, deberían haber investigado en profundidad el nivel de entendimiento y el efecto en la compra de los consumidores para diseñar estrategias específicas de educación nutricional que podrían ser efectivas para complementar la aplicación del ES.

Otro aspecto importante que no se menciona ni se analiza en el manuscrito es la evaluación del objetivo mismo de la regulación del ES que es "garantizar el derecho de las personas a la información oportuna, clara, precisa y no engañosa sobre el contenido y características de estos alimentos". Dentro de la implementación del ES es básico la evaluación del cumplimiento del rotulado en los productos empacados y la concordancia entre el ES y el rotulado tradicional con el contenido de nutrientes que se indica en la parte posterior de los productos. Datos preliminares nuestros indican que existe una discordancia entre estos dos tipos de rotulados en un importante porcentaje de productos. Esta discrepancia es evidente en los tres nutrientes que forman parte del semáforo. Es necesario un comentario por parte de los autores del artículo sobre este tema esencial.

En la sección de Resultados se indica que entre 2014-2015 luego de la implementación del ES aumentó el consumo de confitería, lácteos, bebidas no alcohólicas, jugos procesados, gaseosas y, dentro de las gaseosas, las gaseosas "light" se incrementaron en 47,6\% (litros). Es muy llamativo que la implementación del semáforo que pretende limitar el consumo de productos con altos contenidos de azúcar, potencialmente haya logrado el efecto contrario.
Estos resultados no se discuten en la correspondiente sección del manuscrito por lo que creemos que los autores deben dar una explicación de estos resultados. Por otro lado, actualmente hay estudios que asocian el consumo de bebidas "light", que usualmente utilizan edulcorantes artificiales, con cambios en el microbioma intestinal, lo que podría causar alteraciones metabólicas (2).

Finalmente, los autores señalan la necesidad "de revisar el etiquetado y acompañarlo de medidas complementarias con el fin de revertir la tendencia en el patrón alimentario de la población." Sin embargo, sería indispensable analizar la pertinencia de mantener en el ES únicamente los contenidos de azúcar, grasa y sal a la luz de la evidencia reciente sobre el consumo de estos alimentos y su limitado papel en el desarrollo de las enfermedades crónicas no transmisibles $(3,4)$. Además, se debe también considerar otras posibilidades de rotulado alimentario que sean más comprensivas en la evaluación de la calidad nutricional de los alimentos procesados y que han sido también reconocidas por la Organización Mundial de la Salud, como el Nutri-Score en Francia (5).

Conflictos de interés. Ninguno declarado por los autores.

Declaración. Las opiniones expresadas en este manuscrito son responsabilidad del autor y no reflejan necesariamente los criterios ni la política de la $R P S P / P A J P H$ y /o de la OPS.

Marco Fornasini
Nancy Flores
Manuel E. Baldeón
Centro de Investigación Biomédica
Facultad de Ciencias de la Salud Eugenio Espejo
Universidad Tecnológica Equinoccial
Quito, Ecuador
Paola Carrillo
Universidad de las Américas
Quito, Ecuador

\section{REFERENCIAS}

1. Díaz AA, Veliz PM, Rivas-Mariño G, Vance Mafla C, Martínez Altamirano LM, Vaca Jones C. Etiquetado de alimentos en Ecuador: implementación, resultados y acciones pendientes. Rev Panam Salud Publica. 2017;41:e54.

2. Daly K, Darby AC, Shirazi-Beechey SP. Low calorie sweeteners and gut microbiota. Physiology \& behavior. 2016;164(Pt B):494-500.

3. Dehghan M, Mente A, Zhang X, Swaminathan S, Li W, Mohan V, et al. Associations of fats and carbohydrate intake with cardiovascular disease and mortality in 18 countries from five continents (PURE): a prospective cohort study. Lancet. 390 (10107):2050-2062. Este es un artículo de acceso abierto distribuido bajo los términos de la licencia Creative Commons Attribution-NonCommercial-NoDerivs 3.0 IGO, que permite su uso, distribución y reproducción en cualquier medio, siempre
C. que el trabajo original se cite de la manera adecuada. No se permiten modificaciones a los artículos ni su uso comercial. Al reproducir un artículo no debe haber ningún indicio de que la OPS o el artículo avalan a una organización un producto específico. El uso del logo de la OPS no está permitido. Esta leyenda debe conservarse, junto con la URL original del artículo. 
4. Mente A, O'Donnell M, Rangarajan S, Dagenais G, Lear S, McQueen $\mathrm{M}$, et al. Associations of urinary sodium excretion with cardiovascular events in individuals with and without hypertension: a pooled analysis of data from four studies. Lancet. 2016;388(10043):465-75.
5. Michail N. WHO praises France for "straightforward" nutrition logo [página web]. Disponible en: https://www. foodnavigator.com/Article/2017/03/24/WHO-praises-Francefor-straightforward-nutrition-logo. Acceso el 10 de noviembre de 2017

Forma de citar Fornasini M, Flores N, Carrillo P, Baldeón ME. Etiquetado de alimentos en Ecuador [carta]. Rev Panam Salud Publica. 2018;42:e37. https://doi.org/10.26633/RPSP.2018.37 